\title{
Concepción didáctica para contribuir a la formación del especialista en Turismo hacia su función social
}

Autora: Mabel Font Aranda, PhD.

Universidad Laica Eloy Alfaro de Manabí, Manta, Ecuador (ULEAM).

Contacto: mabel.font@uleam.edu.ec, mabelfont@yahoo.es

RECEPTADO: 18/09/2017

ACEPTADO: 10/11/2017

\section{Resumen}

Ante el reto de un desarrollo turístico sostenible que implique el beneficio, respeto y la participación de la población local, a la vez que satisfaga las expectativas más exquisitas del visitante, y aporte a la economía del país; se exige de nuevos comportamientos, concepciones y sistemas de gestión pública y empresarial privada. En tal contexto, el trabajo tiene como objetivo desarrollar una concepción didáctica que contribuya a la formación del especialista en Turismo para su función social, a través de determinadas asignaturas profesionalizantes en la carrera Ingeniería en Administración de Empresas Turísticas de la Universidad Laica Eloy Alfaro de Manabí (ULEAM). Se evidencian las experiencias obtenidas en el tratamiento de los contenidos afines al proceso de enseñanza aprendizaje de la asignatura Arqueología, Arte y Patrimonio Nacional. Entre los diferentes métodos y técnicas empleados están del nivel teórico: la revisión bibliográfica, análisis y síntesis, inducción y deducción, comparación, modelación; así como del nivel empírico la revisión de documentos oficiales, la observación participante y proyección, el criterio de especialistas, la entrevista; en el marco de la investigación en - de - y con el colectivo. La estructuración y ejecución de una metodología en el proceso de enseñanza aprendizaje de la asignatura citada, permitió los vínculos sistemáticos entre conocimiento teórico y la práctica curricular en el Museo Pacocha “Dr. José Reyes Cedeño”. Facilitó el desarrollo de hábitos, habilidades y de relaciones con la práctica social, en el reordenamiento de la exposición de artefactos y la ejecución de actividades educativas comunitarias, a través del vínculo entre los componentes académico e investigativo.

Palabras clave. Concepción didáctica, especialista en turismo, museo, formación en turismo

Didactic conception to contribute to the training of the Tourism specialist for its social function 


\begin{abstract}
Faced with the challenge of a sustainable tourism development that implies the benefit, respect and participation of the local population, while satisfying the most exquisite expectations of the visitor, and contributing to the economy of the country; it demands of new behaviors, conceptions and systems of public and private business management. The objective of the work is to develop a didactic conception that contributes to the training of the specialist in Tourism for its social function, through certain professionalizing subjects in the career in Tourism Management of the Eloy Alfaro de Manabí Laica University (ULEAM). The experiences obtained in the treatment of contents related to the teachinglearning process of the subject Archeology, Art and National Heritage are evident. Among the different methods and techniques employed are the theoretical level: the bibliographic review, analysis and synthesis, induction and deduction, comparison, modeling; as well as the empirical level, the review of official documents, participant observation and projection, the criteria of specialists, the interview; in the framework of research in - of - and with the collective. The structuring and execution of a methodology in the teaching-learning process of the aforementioned subject, allowed the systematic links between theoretical knowledge and curricular practice in the Pacocha Museum. It allowed the development of habits, skills and relationships with social practice, in the rearrangement of artifact exposition and the development of community educational activities, through the link between academic and research components.
\end{abstract}

Keywords. Didactic conception, specialist in tourism, museum, tourism training 


\section{Introducción.}

Comprender la complejidad que caracteriza el turismo, implica la interpretación de numerosos fenómenos y procesos económicos, sociales y naturales; el menosprecio a uno de ellos conlleva inevitablemente al fallo, de ahí que el especialista en turismo debe comprender e interactuar de forma adecuada con dichos componentes, protegiéndolos en el proceso de prestación del servicio. Una concepción didáctica que contribuya a la formación de este especialista hacia su función social, tiene que estar ineludiblemente vinculada a la organización empresarial y del destino.

En el ámbito referido, el desarrollo de un aprendizaje organizacional es fundamental, al concebirlo como "una actividad social donde el conocimiento y las habilidades se ponen en práctica, se critican y son integradas como oportunidades para optimizar el aprendizaje y la efectividad en el ámbito laboral. Supone un aporte activo de las personas que inician procesos a través del cual exploran y descubren nuevos conocimientos a partir del quehacer cotidiano en la búsqueda de respuestas y soluciones a problemas definidos en conjunto" (Charnes, 2002).

Un reto importante es la preparación del futuro profesional para la gestión desde su formación en pregrado, potencializada por la experiencia práctica en la empresa turística y el cumplimiento de las metas de los presentes paradigmas del aprendizaje organizacional.

La visión fenomenológica expresada, manifiesta peculiar importancia en las condiciones de la República del Ecuador, dado el papel del turismo en el ámbito del cambio de la matriz productiva, en su transición hacia una economía de servicios y conocimiento. Se sustenta en la aprobada Estrategia Nacional (SENPLADES, 2013), que buscó el fortalecimiento del sistema productivo basado en eficiencia e innovación. Se intensificaron esfuerzos, dirigidos entre otros, al desarrollo y fortalecimiento de cadenas productivas (cacao, maricultura, metalmecánica, farmacéutica, turismo, software, etc.)

EL Plan Nacional de Desarrollo 2017-2021, en su Objetivo 9: Garantizar la soberanía y la paz, y posicionar estratégicamente al país en la región y el mundo; plantea en la Política 9.4 Posicionar y potenciar a Ecuador como un país megadiverso, intercultural y multiétnico, desarrollando y fortaleciendo la oferta turística nacional y las industrias culturales; fomentando el turismo receptivo como fuente generadora de divisas y empleo, en un marco de protección del patrimonio natural y cultural. Tres metas 2021, guardan relación con el turismo, referidas a incrementar: el número de 
Rev. SINAPSIS, Edición № 11, Vol. 2, Diciembre 2017

empleos turísticos de 137647 a 202 762, para 2021, el número de turistas de 1,4 millones a 2 millones de personas para 2021 y el ingreso de divisas por concepto de turismo receptor de US\$1,4 mm a US\$ 2,5 mm a 2021 (SENPLADES, 2017).

En consonancia, la Ley Orgánica de Educación Superior (LOES), expresa la necesaria contribución a la transformación de la sociedad, a su estructura social, productiva y ambiental; formando profesionales y académicos con capacidades y conocimientos que respondan a las necesidades del desarrollo nacional y a la construcción de la ciudadanía (Órgano del Gobierno del Ecuador, 2010).

Como reflejo de la LOES, el Modelo Educativo de la Universidad Laica Eloy Alfaro de Manabí, plantea una concepción socio-humanista con enfoques constructivistas, complejidad sistémica, así como los enfoques que direccionan la contextualización de aprendizaje, la ecología de saberes, todo esto con el fin de generar ambientes de aprendizaje acordes con los marcos de pertinencia y la realidad de la Universidad (ULEAM, 2016).

Además, en el Reglamento de Vinculación con la Sociedad de la ULEAM se plantea entre las políticas: fortalecer y ampliar varios programas permanentes y de vinculación con la sociedad, entre los que cita las prácticas pre profesionales y los proyectos de vinculación. Refiere también, fortalecer y contribuir al desarrollo y al emprendimiento socioeconómico con apego al principio de pertinencia, respecto de las necesidades del desarrollo local, zonal, regional y nacional (ULEAM, 2016 a)

En el Perfil de Egreso y en el Análisis del perfil del profesional de la de la Carrera de Ingeniería en Administración de Empresas Turísticas de la ULEAM (ULEAM - Facultad de Hotelería Turismo, FHT-, 2016, 2016 a) se realiza la identificación de las situaciones problemáticas que el profesional enfrenta en su accionar cotidiano y que los docentes utilizarán en el diseño de estrategias de enseñanza y aprendizaje que se desarrollarán en los distintos semestres formativos, en ambos documentos se enfatiza en la articulación de las perspectivas de los mundos del trabajo y de la formación.

El estudiante de Ingeniería en Administración de Empresas Turísticas de la ULEAM, durante la Práctica Curricular puede aprender, sugerir y fungir como auditor, a la vez que incorpora los nuevos desafíos que imponen las actuales transformaciones cualitativas de la Educación Superior.

En el contexto referido el trabajo tiene como objetivo desarrollar una concepción didáctica que contribuya a la formación del especialista en Turismo para su función social, a través de determinadas asignaturas profesionalizantes en la carrera Ingeniería en Administración de Empresas Turísticas de la 
Rev. SINAPSIS, Edición № 11, Vol. 2, Diciembre 2017

Universidad Laica Eloy Alfaro de Manabí. Se evidencian las experiencias obtenidas en el tratamiento de los contenidos afines al proceso de enseñanza aprendizaje de la asignatura Arqueología, Arte y Patrimonio Nacional.

\section{Materiales y métodos}

Para el desarrollo de la concepción didáctica que contribuya a la formación del especialista en Turismo hacia su función social, se siguen diferentes pasos que se muestran en la tabla 1.

Amerita esclarecer, la utilización de determinadas técnicas de la metodología de investigación cualitativa expresada en la obra de Ruiz Olabuénaga (1999)

Captación y reconstrucción de significados: Los métodos cualitativos parten del supuesto básico de que el mundo social es un mundo construido con significados y símbolos, lo que implica la búsqueda de esta construcción y de sus significados, este paradigma adquiere relevancia para la asignatura Arqueología, Arte y Patrimonio Nacional.

Tabla1. Secuencia de pasos para el desarrollo de la concepción didáctica

\begin{tabular}{|l|l|}
\hline Secuencia de pasos & Métodos, técnicas y herramientas \\
\hline $\begin{array}{l}\text { I-Contextualización para la formación } \\
\text { especialista en Turismo hacia su función social. }\end{array}$ & $\begin{array}{l}\text { Revisión de documentos oficiales, analítico } \\
\text { sintético, comparación, modelación esquemática }\end{array}$ \\
\hline II- Estructuración del programa de la asignatura & $\begin{array}{l}\text { Revisión bibliográfica, revisión de documen } \\
\text { oficiales, criterio de especialistas }\end{array}$ \\
\hline $\begin{array}{l}\text { III-Identificación de escenarios óptimos para } \\
\text { desarrollo de las prácticas curriculares. }\end{array}$ & $\begin{array}{l}\text { Observación participante y proyección, inductivg } \\
\text { deductivo, entrevista }\end{array}$ \\
\hline IV-Ejecución del programa & $\begin{array}{l}\text { Captación y reconstrucción de significad } \\
\text { inductivo - deductivo, estudio de } \\
\text { Investigación en - de - y con el colectivo. } \\
\text { (elaboración conjur } \\
\text { Métodos didácticos cos } \\
\text { búsqueda parcial e investigativos) }\end{array}$ \\
\hline V- Control & $\begin{array}{l}\text { Lista de chequeo, control de miembros } \\
\text { VI- Evaluación }\end{array}$ \\
$\begin{array}{l}\text { Taller de exposición in situ. } \\
\text { Evaluación sistemática del proceso de enseñar } \\
\text { aprendizaje (En el programa) }\end{array}$ \\
\hline
\end{tabular}

Fuente. Elaboración propia. 
Rev. SINAPSIS, Edición № 11, Vol. 2, Diciembre 2017

Estudio de Casos: Se emplea cuando cada grupo de estudiantes participa en la resolución de determinada unidad contextual, asociada a una galería en el museo, que transforma positivamente, en el tránsito del análisis teórico, el estado actual descrito, las opciones de cambio y un nuevo resultado.

Investigación en - de - y con el colectivo: tiene como eje central la ejecución de los temas de práctica curricular, donde participan equipos de estudiantes que comparten una misma experiencia, ellos involucran a otros actores y entre todos captan y reconstruyen significados, en el ámbito de este trabajo asociado a un Museo Arqueológico y Etnográfico.

Control de miembros: Mediante examen continúo de datos e interpretaciones con los grupos de estudiantes, especialista y pobladores de los que han sido extraídos los datos.

\section{Resultados}

Los resultados se muestran siguiendo la secuencia de pasos para el desarrollo de la concepción didáctica, expresados en la tabla 1.

\section{1-Contextualización para la formación del especialista en Turismo hacia su función social.}

La contextualización considera aspectos socioeconómicos que transitan desde la mirada nacional hasta la local y aspectos del proceso educativo, vinculados a las políticas ministeriales, de la ULEAM, la Carrera y el proceso didáctico de la asignatura Arqueología, Arte y Patrimonio Nacional. La figura 1, muestra la interpretación esquemática general realizada por la autora. 


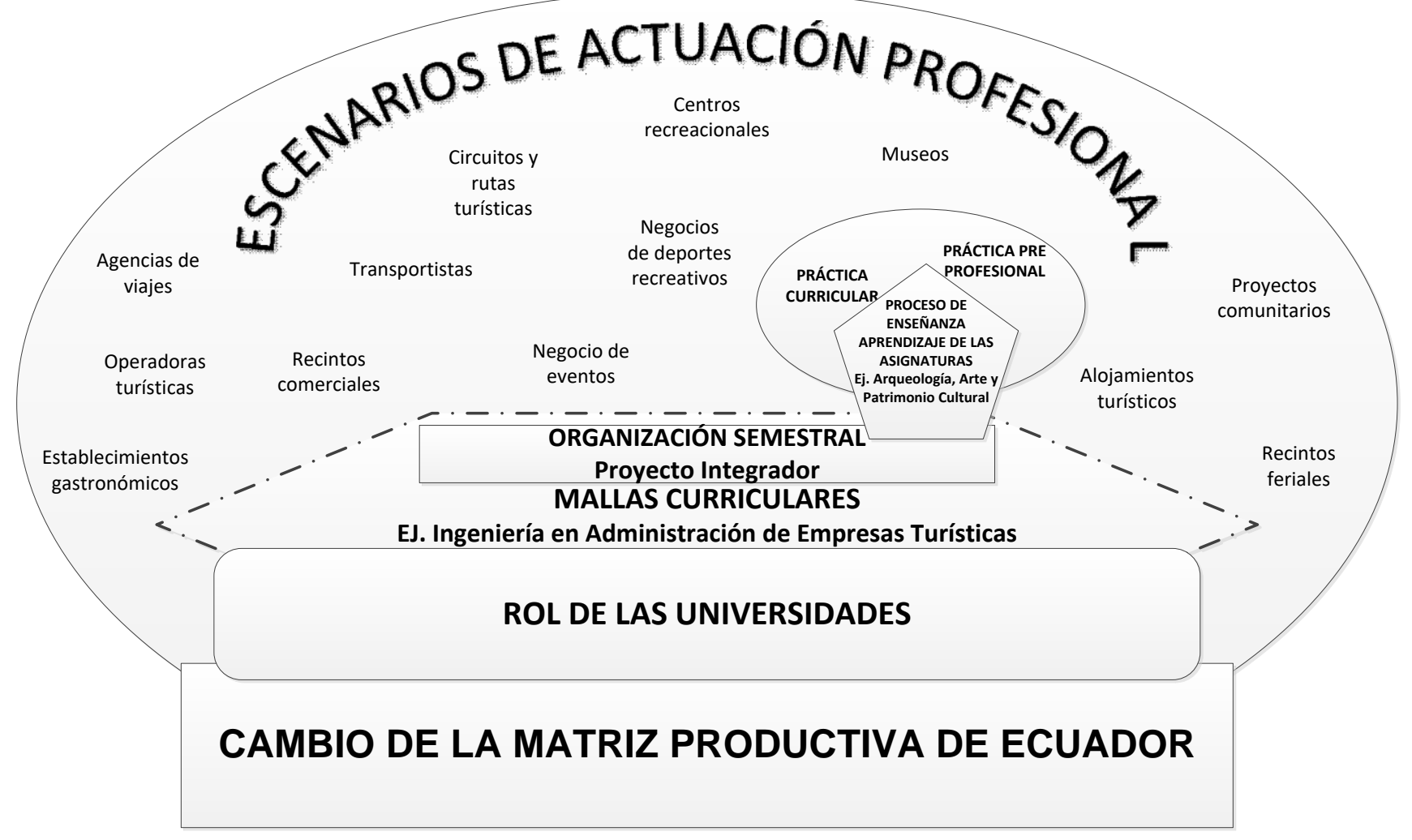

Figura 1. Interpretación esquemática de las condiciones favorables para contribuir a la formación del especialista en Turismo hacia su función social.

Fuente. Elaboración propia.

En la introducción, se hizo alusión a determinados aspectos que de forma sintética, expresan las condiciones favorables en la proyección socioeconómica de Ecuador, para la formación de los especialistas en turismo. También, los vínculos con la educación superior expresada en la LOES, y criterios clave del perfil del profesional de la carrera Ingeniería en Administración de Empresas Turísticas (SENPLADES, 2013, 2017; Órgano del Gobierno del Ecuador, 2010; ULEAM, 2016, 2016a; ULEAM -FHT, 2016, 2016 a).

Desde el punto de vista didáctico la concepción se sostiene en los siguientes principios:

1-Es preciso pasar de la clase magistral disciplinada a formas de organización del Proceso Docente Educativo colaborador, flexible, autónomo, dinámico y participativo (Aprendizaje Significativo y desarrollador). 
Rev. SINAPSIS, Edición № 11, Vol. 2, Diciembre 2017

2-El paradigma tiene que evolucionar hacia metodologías que se centren en el estudiante, que hagan de él un elemento activo en el aprendizaje, con una guía adecuada y adaptada por medio de una tutoría efectiva y comprometida.

3-Realización de la estructuración de las formas organizativas del proceso docente-educativo con énfasis en la actividad independiente, práctica e investigativa del estudiante contextualizadas en su realidad social.

Los principios, constituyen normas que orientan cómo incidir para que el estudiante desarrolle la autonomía, el pensamiento crítico, actitudes colaboradoras y destrezas profesionales, lo que exige nuevas formas de concebir las funciones de la docencia, la investigación y su extensión a la comunidad y al espacio donde pueden tributar como profesionales una vez egresados. Las actuales condiciones de Ecuador, son favorables para que fluyan las configuraciones de formación profesional ya referidas y visualizadas en la figura 1

Los criterios anteriores se sustentan en los posicionamientos del Modelo Educativo ULEAM (2016) respecto a Chaparro (1995: 2), Karlin \& Vianni, (2001), Duarte (2003) y por otra parte en Vygotsky (1896-1934) al considerar al hombre como el resultado del desarrollo histórico y social donde el conocimiento es un proceso de interacción entre el sujeto y el medio no solo físico sino también sociocultural, se enfatiza en la edificación de nuevas cogniciones y maneras de pensar mediante la exploración y la manipulación activa de objetos e ideas, abstractas y concretas; la construcción personal que realiza el hombre en interacción con el mundo circundante.

\section{II- Estructuración del programa de la asignatura}

Para estructurar el programa de Arqueología, Arte y Patrimonio Nacional, se consulta bibliografía de autores como: Ontaneda (2009 y 2010), Órgano del Gobierno del Ecuador. (2004). Ley del Patrimonio Cultural, Echeverría (2011). Mejía (2014), Quinatoa (s/f), y el sitio web del INPC, entre otros.

La revisión del sílabo de la asignatura elaborado por Santos, 2016 y los criterios de especialistas (Tabla 2) 
Tabla 2. Especialistas consultados para la elaboración del programa

\begin{tabular}{|l|l|}
\hline Especialistas consultados & Experiencia \\
\hline Jorge Emilio Santos Toro & $\begin{array}{l}\text { Docente universitario con más de } 30 \text { años de experiencia en mater } \\
\text { afines (Ecuatoriano). }\end{array}$ \\
\hline Libertad Regalado Espinosa & $\begin{array}{l}\text { Docente investigadora universitaria (más de 30 años de experienc } \\
\text { Miembro de la Academia Nacional de Historia. Autora de var } \\
\text { libros (Ecuatoriana). }\end{array}$ \\
\hline Isabel Matilla Blanco & $\begin{array}{l}\text { Suficiencia Investigadora en Antropología (1994), ambas por } \\
\text { UPV/EHU. Misionera en Manabí (Ecuador) durante siete añ } \\
\text { Docente Investigadora Universitaria (Española). }\end{array}$ \\
\hline
\end{tabular}

Elementos del programa Arqueología, Arte y Patrimonio Nacional

Forma parte del eje de Formación Profesional (64 horas)

Sumilla de la asignatura: La asignatura contribuye a la formación de valores y al reconocimiento de las culturas prehispánicas como fuentes del patrimonio cultural actual, destacando los vínculos con la cultura mesoamericana. Enfatiza la diversidad del patrimonio mueble, inmueble, inmaterial, arqueológico y su significado a nivel internacional, nacional, provincial y cantonal. Se relaciona con el perfil de egreso ya que prepara al estudiante para conducir el proceso de interpretación turística en museos, rutas o circuitos y otros escenarios. El proyecto integrador de saberes incorpora los contenidos de esta asignatura junto a otras como Organización de Camping y Guianza.

Objetivo de la asignatura: Desarrollar capacidades cognitivas, socio-afectivas y comunicativas sobre arqueología, arte y patrimonio del Ecuador a través del manejo de diferentes fuentes de información digital e impresas y el reconocimiento de artefactos y vivencias de la realidad que permitan la interpretación turística en diferentes escenarios.

Nivel de impacto y explicación: Es alto porque desarrolla competencias cognitivas, socio-afectivas y comunicativas necesarias para que, desde la Administración turística pública o privada, logre incidir en los procesos de desarrollo económico, social, político, ambiental y cultural del país. 
Contenidos:

\begin{tabular}{|c|c|}
\hline \multicolumn{2}{|r|}{$\begin{array}{l}\text { Unidad } \text { Geografía y cronología de la histo } \\
\text { precolombina andina y de Ecuador ( } 20 \text { horas) } \\
\text { Generalidades sobre la historia precolombina Andina } \\
\text { Vínculos entre la historia prehispánica de Mesoamérica } \\
\text { Andina, características geográficas. } \\
\text { Generalidades sobre la geografía y cronología precolombina } \\
\text { Ecuador. } \\
\text { Periodo Precerámico o Paleoindio y Periodo Formativo } \\
\text { Agroalfarero } \\
\text { Periodo de Desarrollo Regional o Agrominero } \\
\text { Periodo de Integración o Señoríos Étnicos. } \\
\text { Período Inca }\end{array}$} \\
\hline $\begin{array}{l}\text { Unidad 3. Arte y patrimonio de Ecuador (16 horas) } \\
\text { Generalidades sobre arte y patrimonio cultural de Ecuar } \\
\text { Clasificación. Patrimonio mundial de Ecuador. } \\
\text { Patrimonio mueble } \\
\text { Patrimonio inmueble } \\
\text { Patrimonio inmaterial } \\
\text { Patrimonio mueble y arte prehispánico } \\
\text { Patrimonio inmueble y arte Colonial, Republicano } \\
\text { Contemporáneo }\end{array}$ & $\begin{array}{l}\text { Unidad 4. Arqueología, arte y patrimonio regional - lo } \\
\text { (16 horas) } \\
\text { Generalidades sobre la arqueología, arte y patrimonio regior } \\
\text { local } \\
\text { Cultura Manteña Huancavilca } \\
\text { Cultura Jama Coaque } \\
\text { Administración de un museo } \\
\text { Acogida al visitante del museo - Marketing }\end{array}$ \\
\hline
\end{tabular}

Ambiente de aprendizaje: Se asumen los criterios sistematizados en el Modelo Educativo ULEAM (2016): aquel donde los estudiantes interactúan, bajo condiciones óptimas que enriquezca la producción de saberes con el trabajo colaborativo. se instaura en las dinámicas que constituyen los procesos educativos y que involucran acciones, experiencias y vivencias por cada uno de los participantes; actitudes, condiciones materiales y socio-afectivas, múltiples relaciones con el entorno y la infraestructura necesaria para la concreción de los propósitos culturales que se hacen explícitos en toda propuesta educativa (Chaparro 1995: 2) (Duarte, 2003). El constructivismo enfoca al aprendizaje como el resultado de construcciones mentales; esto es, que los seres humanos, aprenden construyendo nuevas ideas o conceptos, en base a conocimientos actuales y previos (Karlin \& Vianni, 2001).

Procesos y estrategias para el componente presencial: Conferencias, clases prácticas, talleres, seminarios donde se realiza manejo de bases de datos y acervos bibliográficos, trabajo en equipos, exposiciones.

Procesos y estrategias para otros escenarios: Comunidades y entidades turísticas donde se realiza el levantamiento de información mediante fichas técnicas, fotografías, videos, otros. Aplicación de técnicas de la profesión en la transferencia de información turística y elaboración de guiones. 
Rev. SINAPSIS, Edición № 11, Vol. 2, Diciembre 2017

Procesos y estrategias para el componente autónomo: Análisis y comprensión de materiales bibliográficos y documentales, tanto analógicos como digitales.

Procedimientos de evaluación:

Tabla 3. Estrategias de evaluación.

\begin{tabular}{|c|c|c|}
\hline Ámbito & Pes & Estrategias \\
\hline \multirow[t]{4}{*}{ Actuación } & \multirow[t]{4}{*}{309} & Aplicación de un cuestionario sobre Tutela y protección del patrimonio arqueológico. \\
\hline & & $\begin{array}{l}\text { Participación en exposición grupal sobre los periodos de la historia precolombina } \\
\text { Ecuador. }\end{array}$ \\
\hline & & Video debate patrimonio inmaterial y artes escénicas y folclor. \\
\hline & & Exposición grupal sobre patrimonios de la provincia de Manabí y cantón Manta. \\
\hline \multirow[t]{8}{*}{$\begin{array}{l}\text { Producción } \\
40 \%\end{array}$} & \multirow[t]{4}{*}{209} & $\begin{array}{l}\text { Trabajos de observación dirigida en un museo sobre turismo y difusión del patrimo } \\
\text { cultural. }\end{array}$ \\
\hline & & $\begin{array}{l}\text { Manejo de base de datos y acervos bibliográficos de la Biblioteca del Museo Cent } \\
\text { (Informe de visita) }\end{array}$ \\
\hline & & $\begin{array}{l}\text { Elaboración, aplicación y análisis de guía de observación sobre patrimonio inmueble y a } \\
\text { Colonial, Republicano y Contemporáneo en Manta. }\end{array}$ \\
\hline & & Aplicación e interpretación fichas técnicas \\
\hline & \multirow[t]{4}{*}{200} & Resolución de problemas o casos sobre turismo y difusión del patrimonio cultural. \\
\hline & & $\begin{array}{l}\text { Resolución de problemas sobre geografía y cronología precolombina de Ecuador. (Cua } \\
\text { sinóptico) }\end{array}$ \\
\hline & & Manejo de base de datos Web INPC. \\
\hline & & Selección y preparación de Ficha técnica sobre Administración de un museo. \\
\hline \multirow[t]{2}{*}{ Acreditació } & \multirow[t]{2}{*}{309} & Prueba escrita \\
\hline & & Informe de práctica curricular \\
\hline
\end{tabular}

Fuente. Elaboración propia a partir del modelo Vicerrectoría Docente ULEAM, 2017.

\section{III-Identificación de escenarios óptimos para el desarrollo de las prácticas curriculares}

La selección de los escenarios de práctica curricular, parte de los convenios institucionales establecidos por la Universidad y en particular con la Facultad de Hotelería y Turismo. En el ámbito de la asignatura Arqueología, Arte y Patrimonio Nacional, los museos constituyen recintos excelentes para contrastar la teoría con la práctica, por que albergan numerosos artefactos arqueológicos, objetos y documentos del patrimonio cultural, además en ellos tienen lugar diversas funciones sociales, donde el especialista en turismo encuentra lugar para su actuación profesional.

En correspondencia con los supuestos referidos, se selecciona como escenario óptimo para la práctica curricular, el Museo Pacocha, varias razones justifican la decisión son:

1- Reapertura el 20 de mayo de 2017, coincidiendo con el inicio del semestre. 
Rev. SINAPSIS, Edición № 11, Vol. 2, Diciembre 2017

2- Génesis del proyecto: por petición de los moradores de la comuna rural de Pacoche y bajo convenio marco de cooperación interinstitucional entre las autoridades de la ULEAM, el Gobierno Autónomo Descentralizado de Manta y Parroquial de Santa Marianita.

3- Todos los documentos y artefactos que pertenecían al museo destruido durante el terremoto del 16 de abril y ubicado en la Universidad, pasaron a conformar la exposición en la nueva edificación.

4- En este lugar, antes (finales de la década del 90) en una construcción deteriorada con el pasar de los años, se colocaron los primeros artefactos arqueológicos obtenidos de la misma localidad.

5- El museo se ubica a $26 \mathrm{~km}$ de Manta, en una zona tropical privilegiada, a 10,4 km (15 min) de la entrada oficial del Refugio de Vida Silvestre Marino Costera Pacoche. Se destaca la combinación de la naturaleza y el ambiente rural, rico en cultura, vestigios arqueológicos in situ y superficiales, donde el montuvio tiene diferentes crías de animales y numerosos espacios para descansar o practicar actividades al aire libre.

De la entrevista realizada al MSc. Pedro Montalván Acosta (Coordinador del Museo) e Ing. Fátima Reyes Reyes (Secretaria), se advierte el interés por constituir el Museo Pacocha en un laboratorio donde los estudiantes universitarios realizan sus prácticas, entre ellas la de Arqueología, arte y Patrimonio Nacional. Posteriormente se consensuó el sistema de actividades y los grupos de estudiantes (Tabla 4). Para el logro de los resultados se armonizan las actividades de Docencia y de las prácticas de Aplicación y Experimentación de Aprendizajes con la Práctica Curricular que se organizó en diferentes temas a abordar por grupos de cinco estudiantes en el ámbito del museo Pachocha y la comunidad que lo circunda. Se utilizó la investigación acción participativa para incidir en práctica profesional y la vinculación con la sociedad.

Tabla 4. Aspectos cruciales a abordar por grupos de estudiantes

\section{Temas y equipos}

1-Reordenamiento del Museo Pachocha

-Diagnóstico

-Estudio de la competencia. Qué ofertan otros museos.

-Cómo mantener la diversidad de la exposición pero diferenciándose.

-Qué es lo óptimo para el Museo.

2- Memorias sobre la evolución de la fundación del museo.

-Identificación de personas conocedoras de la comunidad.

-Revisión de fuentes bibliográficas históricas.

-Elaboración de un documento explícito e ilustrativo sobre el tema.

-Elaboración de una placa de madera, propia de la zona con los aspectos relevantes a la fundación del museo.

3- Reordenamiento de la Galería de Arqueología

-Diagnóstico de los artefactos arqueológicos exhibidos. 
- Diagnóstico de los artefactos arqueológicos en bodega.

-Selección de los artefactos arqueológicos más representativos y mejor conservados.

-Cedulación de los artefactos seleccionados.

- Organización de la exposición

4- Unificar Galería Colonial y Republicana, Fotografía del Ferrocarril de Manabí, Biografía, Biográfica y Fotográfica

General José Eloy Alfaro Delgado y Galería Musical.

-Diagnóstico de las galerías

-Determinación de una lógica de organización.

-Selección de los objetos, materiales, documentos más representativos y mejor conservados.

-Cedulación de los objetos, materiales, documentos, etc.

- Organización de la exposición

5- Reordenamiento de la Galería de Etnografía

-Diagnóstico de los objetos exhibidos.

- Diagnóstico de los objetos en bodega.

-Selección de los objetos más representativos y mejor conservados.

-Cedulación de los objetos seleccionados.

6- Proyecto Manta Rural

Diseño de un proyecto sobre investigación local rural y vinculación con la comunidad. Se empleará el formato

Vinculación de la ULEAM.

7- Función educativa del Museo Pacocha

-Elaboración de guiones de actividades a desarrollar con la comunidad.

- Consideración de los grupos niños, jóvenes, adultos mayores, etc.

-Desarrollo de experiencias como concursos, curso de educación no formal, etc.

\section{IV-Ejecución del programa}

Se desarrolló el total de las sesiones y actividades planificadas en la asignatura, las formas de organización del proceso de enseñanza aprendizaje predominantes, exigieron la participación de los estudiantes de manera activa. Los diferentes museos de la ciudad de Manta, se convirtieron en escenarios de conocimientos y de contrastación de la teoría con la práctica.

La práctica curricular fue concluida por cinco de los siete grupos, aquellos que fueron sistemáticos desde el inicio del semestre, lograron cumplir las orientaciones con excelentes resultados y contribuyeron a la mejora de la exposición en el Museo Pacocha, o desarrollaron actividades con diferentes personas de la comunidad. Algunas evidencias

Fragmento del informe del equipo 5. Reordenamiento de la Galería de Etnografía 
En el presente informe se reflejan los resultados de la práctica curricular de la materia de Arqueología, Arte y Patrimonio Nacional, en el cual se detalla cómo se procedió para la planificación del trabajo con los integrantes del grupo, luego se realizó una investigación a fondo de cómo esta ordenado y cedulado el Museo Municipal Etnográfico Cancebí en la ciudad de Manta, como base para la organización de la Galería Etnográfica de este Museo y luego proceder a la cedulación.

Hicimos un diagnóstico de los objetos exhibidos en la Galería Etnográfica del Museo Pacocha para identificar los elementos mejor conservados y los que no están bien conservados se procedió a retirarlos de la galería. En el presente informe se detallan cuáles fueron los artefactos etnográficos que se retiraron. También se hizo un diagnóstico de los objetos de etnografía que se encontraban en bodega, de los cuales se hizo una lista de aquellos que deberían estar en exhibición en dicha galería. Se reordenó la exposición, con el siguiente orden: primero la casa típica manabita, segundo las manufacturas hechas a mano, tercero las herramientas de trabajo y en la última parte los utensilios de cocina y utensilios hechos con mate, finalmente se culminó el trabajo cedulando cada uno de los objetos.

Las siguientes fotografías evidencian momentos de la ejecución del programa.
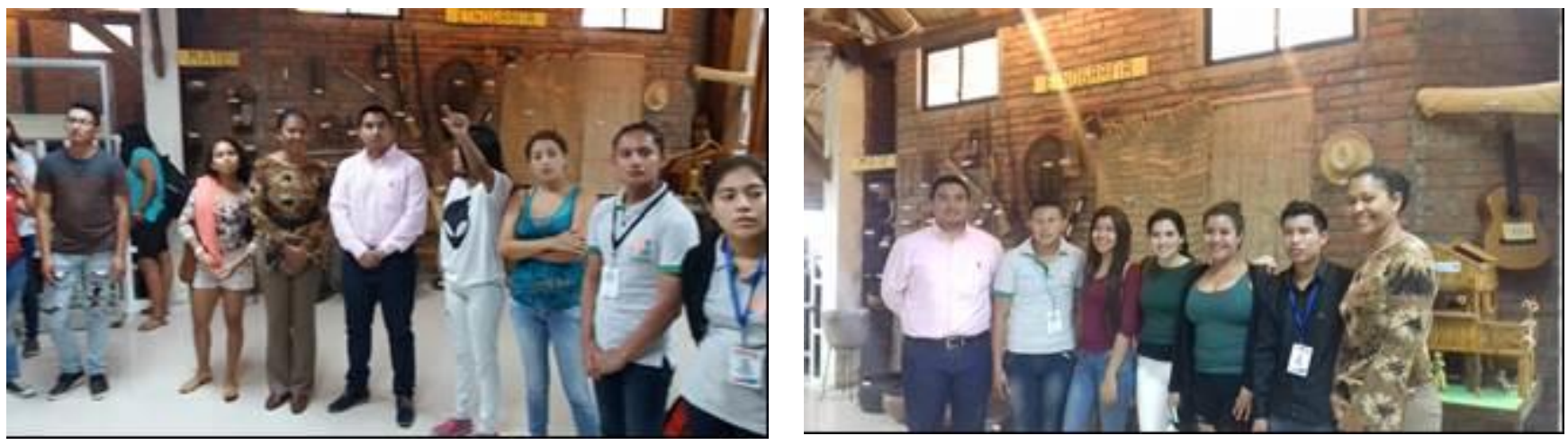

Figura 2. Reordenamiento de galerías

Durante el proceso de desarrollo del programa en el semestre siguiente, cuando los estudiantes constataban la tutela y protección del patrimonio cultural en el Museo Pacocha, identificaron la Galería Etnográfica como la mejor organizada.

\section{V- Control}

La principal vía de control fue el registro sistemático de la asistencia a las actividades docentes, y el llenado de las planillas normadas para las prácticas curriculares, además se realizó el chequeo de la evolución de las acciones desarrolladas por los estudiantes. Cinco de los siete equipos, asistieron sistemáticamente a las prácticas curriculares y resolvieron las actividades semanalmente. Dos grupos 


\section{Rev. SINAPSIS, Edición № 11, Vol. 2, Diciembre 2017}

de estudiantes no lograron obtener los resultados deseados e incumplieron las acciones programadas, pues aunque se efectuó la orientación de las actividades docentes y de la práctica curricular desde el inicio del semestre, estos subvaloraron el nivel de complejidad de las tareas, y las relegaron hasta el final del semestre. La alerta permanente de la docente y el chequeo de la resolución de las tareas fue efectivo en la mayoría, pero no en todos.

\section{VI- Evaluación}

El proceso de evaluación fue frecuente, y mediante retroalimentación profesora - estudiantes y con los especialistas del Museo. El criterio evaluativo final, dependió de la exposición de los resultados en la galería transformada o en el contexto de la comunidad. El taller de exposición in situ, permitió el debate y el intercambio de criterios, el planteamiento de los logros o resoluciones pendientes. Las fotografías evidencian el taller de exposición in situ.
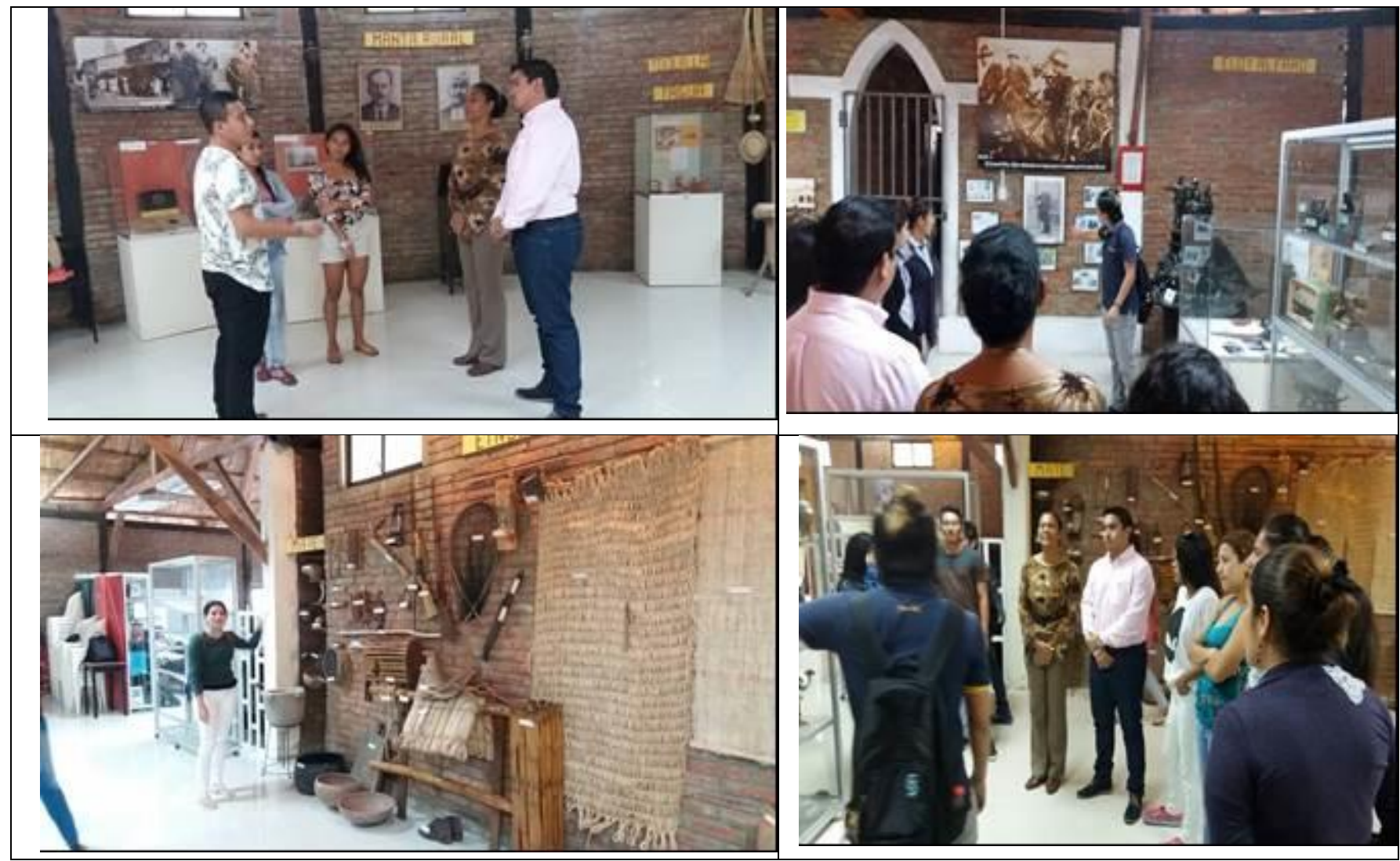

Figura 3. Taller de exposición in situ

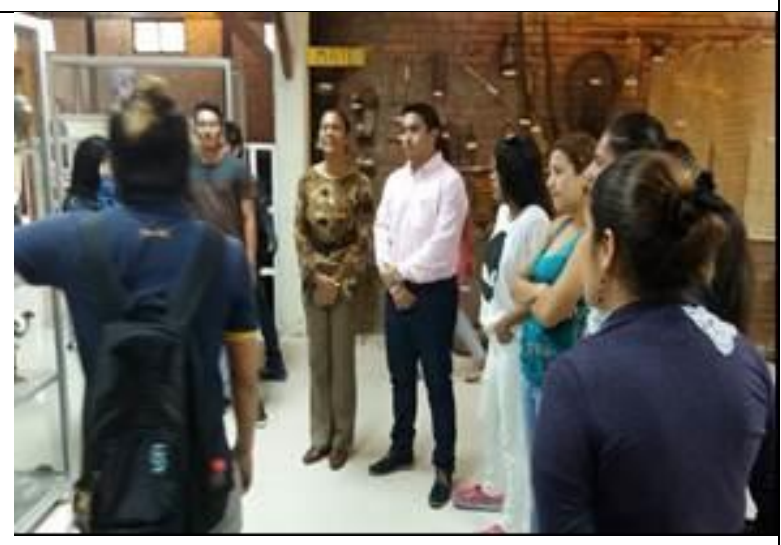


Rev. SINAPSIS, Edición № 11, Vol. 2, Diciembre 2017

\section{Discusión}

Quedó demostrada la viabilidad del desarrollo de una concepción didáctica para contribuir a la formación del especialista en Turismo hacia su función social, a través de la experiencia en la asignatura Arqueología, Arte y Patrimonio Nacional, por lo que se cumple el objetivo planteado.

Se corrobora el planteamiento Charnes (2002) respecto al aporte activo de los estudiantes que iniciaron procesos de investigación acción en el Museo Pacocha, a través del cual exploraron y descubrieron nuevos conocimientos, en la búsqueda de respuestas y soluciones a problemas definidos en conjunto, como fue el reordenamiento de algunas galerías.

El desarrollo de la concepción didáctica propuesta está en armonía y aporta al objetivo 9 del Plan Nacional de Desarrollo 2017-2021 (SENPLADES, 2017), siendo reflejo de la Loes, el Modelo Educativo y el Reglamento de Vinculación con la Sociedad de la Universidad Laica Eloy Alfaro de Manabí (ULEAM, 2016 - 2016 a).

Por otra parte, en la concepción didáctica presentada, se utilizan estrategias que tributan al perfil profesional del Ingeniero en Administración de Empresas Turísticas. Además, favorece a que el estudiante desarrolle la autonomía, el pensamiento crítico, actitudes colaboradoras y destrezas profesionales, como señala el Modelo Educativo ULEAM (2016) respecto a Chaparro (1995: 2), Karlin \& Vianni, (2001), Duarte (2003) y por otra parte en Vygotsky (1896-1934)

Mediante esta concepción los contenidos sistematizados por los autores Ontaneda (2009 y 2010), Órgano del Gobierno del Ecuador. (2004). Ley del Patrimonio Cultural, Echeverría (2011). Mejía (2014), Quinatoa (s/f), y el sitio web del INPC, son constatados por los estudiantes en la práctica curricular.

Los estudiantes no siempre valoran positivamente las exigencias y la presión que exige la configuración del programa, estiman excesivo el cúmulo de actividades que se les orienta, no obstante los que cumplen las metas requeridas, manifiestan satisfacción y orgullo por las mejoras incorporadas. En este particular, se hace necesario añadir alternativas para modificar los modos de actuación que obstruyen el proceso de formación profesional. 
Rev. SINAPSIS, Edición № 11, Vol. 2, Diciembre 2017

\section{Conclusiones}

La Concepción didáctica para contribuir a la formación del especialista en Turismo hacia su función social, evidencia la viabilidad de su aplicación en el contexto de la asignatura Arqueología, Arte y Patrimonio Nacional, y es factible aplicar a otras, ya que se sustenta en la lógica del Modelo Educativo de la ULEAM y es consecuente con las políticas rectoras del Ecuador.

La experiencia desarrollada evidenció los procedimientos a seguir para lograr el vínculo entre investigación, docencia y vinculación con la práctica turística en el marco de los contenidos de la asignatura Arqueología, Arte y Patrimonio Nacional, en el contexto del Museo Pacocha como con uno de los escenarios de actuación profesional de los estudiantes de Ingeniería en Administración de Empresas Turísticas.

\section{Bibliografía.}

Charnes G. (2002). Aprendizaje organizacional. Llevando las ideas a la práctica. Universidad de Chile, Diplomado en Especialización en Capacitación y Desarrollo, Universidad de Santiago.

Echeverría Almeida, J. (2011). Glosario de arqueología y temas afines. Tomos I y II. INPC. Ediecuatorial. Quito.

FHT- ULEAM (2016). Análisis del perfil profesional Carrera de Turismo 2016. Manta, Ecuador.

FHT- ULEAM (2016 a). Perfil de Egreso Carrera de Turismo 2016. Manta, Ecuador.

Mejía Salazar, A. R. (2014). FORO El patrimonio cultural como derecho: el caso ecuatoriano. Revista de Derecho, n. ${ }^{\text {o } 21, ~ U A S B-E c u a d o r ~ / ~ C E N ~ • ~ Q u i t o . ~}$

Ontaneda Luciano, Santiago. 2009. Ecuador: Hitos de su pasado precolombino Libro. Banco Central del Ecuador. Quito.

Ontaneda Luciano, Santiago. 2010. Las antiguas sociedades precolombinas del Ecuador. Un recorrido por la Sala de Arqueología del Museo Nacional. Banco Central del Ecuador. Quito.

Órgano del Gobierno del Ecuador. (2004). Ley del Patrimonio Cultural. Registro Oficial Suplemento 465 de 19-nov- Quito, Ecuador. Recuperado de: http://www.patrimonio.quito.gob.ec/images/lotaip2015/enero/ley_patrimonio_cultural.pdf 
Rev. SINAPSIS, Edición № 11, Vol. 2, Diciembre 2017

Órgano del Gobierno del Ecuador. (2010). Ley Orgánica de Educación Superior, LOES. Registro Oficial No 298. Quito, Ecuador.

Quinatoa Cotacachi, E. (s/f). Culturas ancestrales ecuatorianas. Universidad Tecnológica Equinoccial.

Recuperado de: http://app.ute.edu.ec/content/3298-369-9-1-18-

10/HISTORIA\%20ABORIGEN\%

20Y\%20FOLKLORE\%20ECUATORIANO.pdf

Ruiz Olabuénaga, J. I. (1999) Metodología de la investigación cualitativa. 2. a edición. Universidad de Deusto. Bilbao. P. 247.

Secretaría Nacional de Planificación y Desarrollo (SENPLADES) (2013). Plan Nacional de Desarrollo / Plan Nacional para el Buen Vivir 2013-2017. Disponible en www.buenvivir.gob.ec, Quito: Autor.

Secretaría Nacional de Planificación y Desarrollo (SENPLADES). (2017). Plan Nacional de Desarrollo 2017-2021. Toda una Vida. Quito - Ecuador http://www.planificacion.gob.ec/wpcontent/uploads/downloads/2017/10/PNBV-26-OCTFINAL_0K.compressed1.pdf

ULEAM (2016). Modelo educativo de la ULEAM. Vicerrectorado académico. Imprenta Universitaria. Manta, Ecuador.

ULEAM (2016a). Reglamento de Vinculación con la Sociedad de la ULEAM. Manta, Ecuador. 